\title{
Improved Pattern Recognition Classification Accuracy for Surface Myoelectric Signals using Spectral Enhancement
}

\author{
Paul McCool, Lykourgos Petropoulakis, John J. Soraghan Senior Member, IEEE and Navin Chatlani
}

\begin{abstract}
In this paper, we demonstrate that Spectral Enhancement techniques can be configured to improve the classification accuracy of a pattern recognition-based myoelectric control system. This is based on the observation that, when the subject is at rest, the power in EMG recordings drops to levels characteristic of the noise. Two Minimum Statistics techniques, which were developed for speech processing, are compared against electromyographic (EMG) de-noising methods such as wavelets and Empirical Mode Decomposition. In the cases of simulated EMG signals contaminated with white noise and for real EMG signals with added and intrinsic noise the gesture classification accuracy was shown to increase. The mean improvement in the classification accuracy is greatest when Improved Minima-Controlled Recursive Averaging (IMCRA)-based Spectral Enhancement is applied, thus demonstrating the potential of Spectral Enhancement techniques for improving the performance of pattern recognition-based myoelectric control.
\end{abstract}

Index Terms—electromyography, myoelectric, spectral enhancement, IMCRA

\section{INTRODUCTION}

Surface Electromyography (EMG) is a non-invasive measurement method of muscle activity that can be used for telehealth

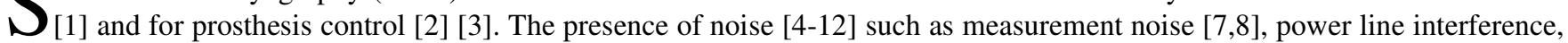
quantisation noise, ECG and motion artifact [4,5] obscure the information content of the signal and reduce its usefulness for pattern recognition-based prosthetic control by causing a reduction in gesture classification accuracy.

Some types of noise can be preventatively reduced by careful hardware setup: for example, Motion Artifact can be reduced by minimising the sensor movement relative to the skin. Once digitized, band pass filtering is used to restrict the frequency content to the band within which most of the energy of the EMG resides. Notch filtering or adaptive filtering [5] blind rejection [9] or spectral interpolation [10] can also be performed to remove $50 \mathrm{~Hz}$ or $60 \mathrm{~Hz}$ power line interference. ECG can be removed by applying template methods or moving average filtering [11].

Noise detection and identification methods have been applied to EMG signals in order to mitigate the effects of noise that could not be preventatively removed. The methods were tested by artificially adding noise to EMG signals. ECG, motion artifact, Additive White Gaussian Noise (AWGN), amplifier saturation and power line interference were added to EMG in [4-6] to test the feasibility of pattern recognition. AWGN was used in [12] to assess the robustness of features and to assess noise reduction techniques such as wavelets in [13,14].

In this paper, we will demonstrate how two spectral enhancement techniques designed for speech signals can be configured for EMG to improve the classification accuracy of pattern recognition: Minimum Statistics Noise Estimation (MSNE) and Improved Minima Controlled Recursive Averaging (IMCRA). The techniques are tested with simulated EMG, real EMG that is artificially contaminated with AWGN and an intrinsically noisy EMG data set. To assess the effectiveness of the noise reduction, classification accuracy is used as a means of evaluating EMG signal quality: It is asserted here that if signal quality has been improved, then classification accuracy will increase. Three measurements for evaluating EMG signal quality are first examined, which are assessed on the 'steady state' parts of the EMG. They are:

a. Maximum Drop in Power Density (DP Ratio) is a measurement between the maximum and minimum energy content of FFT bins. The threshold value from [15] is $30 \mathrm{~dB}$.

Part of this work was funded by internal University of Strathclyde research programmes Strathclyde Links and MedTech.

Paul McCool (corresponding author) is with the School of Engineering and Physical Sciences, Heriot-Watt University, Edinburgh, EH14 4AS; (email: p.mccool@hw.ac.uk)

Lykourgos Petropoulakis and John Soraghan are with the Centre for Excellence in Signal and Image Processing, Department of Electronic and Electrical Engineering, University of Strathclyde, George Street, Glasgow, G1 1XW, UK. (phone: +44-141-548-2935; e-mails \{1.petropoulakis, j.soraghan\}@ strath.ac.uk).

Navin Chatlani is with Intel, 1110 American Parkway NE, Allentown, PA 18109 (e-mail: navin.chatlani@gmail.com). 
b. The SN Ratio is a type of SNR in which the shape of the spectrum is taken into account. It is calculated based on the assumption that no EMG is present in the upper $20 \%$ of the frequency range. For our data sets, which will be discussed in 2.1 .2 , the upper $20 \%$ is $800-1000 \mathrm{~Hz}$ and $720-800 \mathrm{~Hz}$. According to [16] surface EMG resides in the band $10-400 \mathrm{~Hz}$ so this assumption is valid. The threshold value for SN Ratio from [15] is $15 \mathrm{~dB}$.

c. The $\Omega$ Ratio is an "index of spectral deformation" [15] used to detect "disturbances" in the EMG spectrum. The threshold value from [15] is 1.4 .

The only prior instance of the application of spectral enhancement to EMG was spectral subtraction in [17], where the mean spectrum of the noise is calculated across several 'noise only' STFT windows to account for its variation. The mean noise spectrum is then subtracted across the STFT windows of the entire signal. However, the authors were unable to find any prior research in which Spectral Enhancement using minimum statistics was applied within the EMG frequency band as a means of noise identification or reduction.

The organization of this paper is as follows: myoelectric signals and the data sets used in the work are introduced in Section 2, EMG filtering is described in Section 3, pattern recognition is described in 2.3, method in 3, results in 4, discussion in 5 and conclusions are given in 6.

\section{THEORY}

\subsection{Surface Myoelectric Signals}

To move the forearm or hand, nerve impulses travel down the nerve to motoneurons, which interface with the muscles at motor units. When the motor units are activated, they fire and cause a potential difference. The activity from single motor unit action potentials (MUAPs) can be detected by invasive needle sensors, or EMG from a group of MUAPs can be sensed by an electrode on the skin's surface. When detected using the latter, the attenuated summation of MUAPs within range of the sensor is called the surface EMG, or sEMG, signal [18]:

$y[n]=\sum_{i=1}^{R} \sum_{l=-\infty}^{+\infty} x_{i l}\left[n-\Phi_{i, l}\right]+v[n]$

$Y[n]$ is the measured SEMG signal, $R$ is the number of active motor units, $x_{i k}[n]$ is $l$ th motor unit action potential belonging to motor unit $i, \Phi_{i, l}$ is the occurrence time of $x_{i l}[n]$ and $v[n]$ is additive noise [18]. The STFT of this is the summation of the STFT of the EMG and additive noise.

$Y[f, t]=X[f, t]+V[f, t]$

\subsubsection{Simulated EMG}

Simulated EMG has mathematical or structural properties similar in some useful way to real EMG. It is guaranteed to be clean, so it is suitable for assessing the performance of spectral noise estimators because the exact amount of additive noise can be controlled. The method from [19] was used, which is a phenomenological model [18] that we previously used in [4,6]. The following transfer function was used to generate simulated EMG [19]:

$H_{E M G}(f)=\frac{j K f_{h}^{2} f}{\left(f_{l}+j f\right)\left(f_{h}+j f\right)^{2}}$

The parameters $f_{l}$ and $f_{h}$ are used to adjust the shape of the EMG spectrum. In this work, the parameters were changed randomly for each simulated gesture, but kept constant during a gesture to simulate a static contraction. A simulated recording of a 'rest-gesture-rest' was generated of length 15 seconds that has a gesture of length $5 \pm 0.5 \mathrm{~s}$ in duration starting between $5-10 \mathrm{~s}$ into the simulated recording. An onset and offset were generated by 100 -sample 'ramps' at either side of the simulated steadystate contraction [20]. Finally, Additive White Gaussian Noise (AWGN) was added to the signal at the required SNR. Fig. 1 (left) shows a clean EMG gesture, which has AWGN added (Fig. 1 (centre)) to produce the signal shown in Fig. 1 (right). 

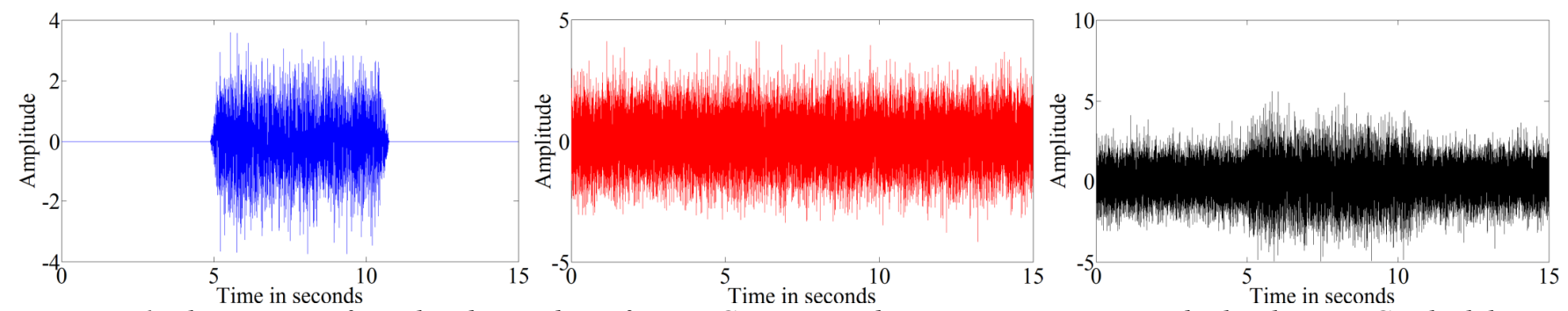

Fig. 1. The creation of simulated recording of an EMG corresponding to a gesture is created. The clean EMG, which has well-defined onset, steady-state and offset locations (left) has noise (centre) added to it (right). In this example, $f_{l}=59, f_{h}=129$, $f_{s}=2000, S N R=O d B . \quad y$-axis units are arbitrary

The parameter $f_{l}$ was chosen randomly from the frequency ranges from $30-60 \mathrm{~Hz}$ and $f_{h}$ was randomly $30-100 \mathrm{~Hz}$ greater than $f_{l}$. The gain factor $K$ was adjusted to make the EMG power unity.

\subsubsection{Real EMG data sets}

Two data sets of real EMG were used in this work. Data Set 1 consists of thirty subjects and six different gesture classes (plus rest) each for four sessions of six trials. Details can be found in [21]. There are eight bipolar channels, of which the seven lowerarm channels were used. The bandwidth of the amplifier was $1 \mathrm{~Hz}-1 \mathrm{kHz}$ and the data set had been provided with a $60 \mathrm{~Hz}$ notch filter applied [21].

Data Set 2 is noisy 16-channel data from five subjects. There are two sessions for each subject, each consisting of a recording with 60 gestures. When used in the context of pattern recognition, one session was used for training and one for testing. There were twelve different gestures: all fingers flexion and extension (including thumb), as well as thumb opposition and antiopposition. Each gesture was initiated from rest and executed in random order.

\subsection{EMG filtering}

EMG can be measured using high-density sensor arrays [22]. Sensors that either degrade or do not contribute significantly to classification accuracy can be discarded through a process such as Sequential Forward Selection [22]. Such spatial filtering cannot be used for the current generation of clinical prosthetics because the number of sensors is limited due to the sensor size, lack of available skin area and limited processing power.

IIR or FIR band pass filters are commonly used and effective at removing noise outside the frequency range within which surface EMG is normally present [3]. Adaptive noise cancellation, which can be used in speech, requires a noise reference signal, and this is unavailable in prosthetic control. Wiener filtering, which also requires a clean reference [23] assumes that the spectrum of the noise is stationary.

Noise reduction was implemented using wavelets in [13] [14]. In this work, the Daubechies db2 with four levels of decomposition from [13] was used with a hard threshold. Like EMD (discussed below), this method is applied to windows of the signal just prior to feature extraction, in contrast to the other methods where the filtering is applied to the entire signal before it is split into windows for feature extraction.

EMD is a method of decomposing a signal into orthogonal Intrinsic Mode Functions (IMFs) that are derived from the signal itself (i.e. not from basis functions as in wavelet transforms) [24]. In [25], the first three IMFs are summed to produce a noisereduced signal. In [26], each IMF has its standard deviation applied as a threshold, and then the signal is reconstituted. Both of these methods are used for comparison in this work.

\subsection{Pattern recognition}

Modern clinical myoelectric control systems, such as those used for hand prostheses, obtain control instructions from a averaged rectified value of myoelectric signals from two sites on the forearm - one on the flexor group and one on the extensor group [2]. This is interpreted into open and close signals for the hand. If the prosthetic has individually articulated fingers, additional gestures can be realized by clever re-use of the open/close signals, for example, a second 'open' signal held when the hand is already open could be used to initiate a 'point' gesture. This requires training and practice on the part of the user, as the muscle movements and desired gestures do not intuitively match [3].

Pattern recognition allows control using muscle movements that correspond more naturally to the intended prosthetic gesture [2]. Many such control systems have been demonstrated in the laboratory environment [27]. In order to extract more information from the signals, features are extracted and combined, along with class labels, for the supervised learning of a classifier such as a neural network or Linear Discriminant Analysis. There are manifold feature types, but the most common, against which novel features are often compared, are time domain features such as the Hudgins' feature set [2]. 


\section{METHOD}

A diagram of the system structure is shown in Fig. 2.

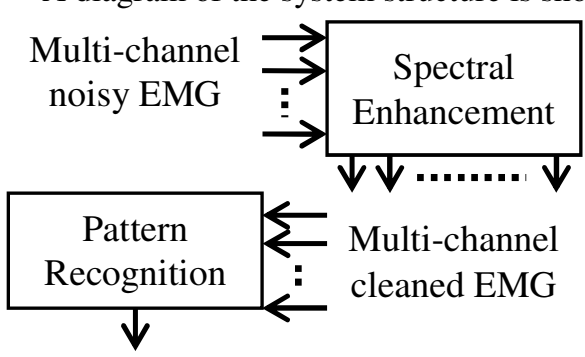

Class labels

Fig. 2 - EMG is pre-processed using one of the Spectral Enhancement methods, and then pattern recognition is performed

Spectral Subtraction is a well-known technique for speech signals in which a spectral estimate of the noise is subtracted from the signal [28]. Minimum Statistics (MS) takes advantage of the fact that during speech the spectral magnitude frequently drops to the noise floor [29]. In each STFT frequency bin, the minimum spectral magnitude tracked over $D$ frames is assumed to be the noise floor. For window $t$ of STFT bin $k$ :

$\min (X[t, k], X[t-1, k] \ldots X[t-D, k])$

Once a spectral noise estimate has been generated, the magnitudes from the STFT noise estimation can be subtracted from the magnitudes of STFT of the original signal to produce a spectrally enhanced signal. Minimum Statistics can be improved by using recursive averaging. Improved Minima Controlled Recursive Averaging (IMCRA), which is based on minimum statistics, was designed to estimate noise in speech through recursive noise estimation on the signal [30] [31]. A noise estimate is made in each time-frequency slot in the form of a measure of the absolute value of the magnitude of the noise power, based on assumptions about the statistical distribution of signal and noise. Once the noise estimate is made, it can be subtracted from the original noisy signal to produce a noise-reduced signal. This step is called IMCRA-based spectral enhancement in this paper, but has also been known as IMCRA/OMLSA [32].

To obtain the noise estimate, the STFT of the signal is smoothed, and then stronger speech components are eliminated from a second, conditional, smoothing. To do this, IMCRA tracks the minima across the signal for each FFT frequency bin. The power in the signal during the rest periods drops to levels characteristic of the noise floor, and the minima should show this.

AWGN contains noise at all frequencies, so it is useful for assessing the performance of noise reduction algorithms across frequency and time. AWGN was added to channel 6 (a dorsal site near the lateral epicondyle) from a recording from Data Set 1. The result is shown in Fig. 3. The movement sequence is hand close; wrist extension; wrist flexion; wrist extension; rest. 


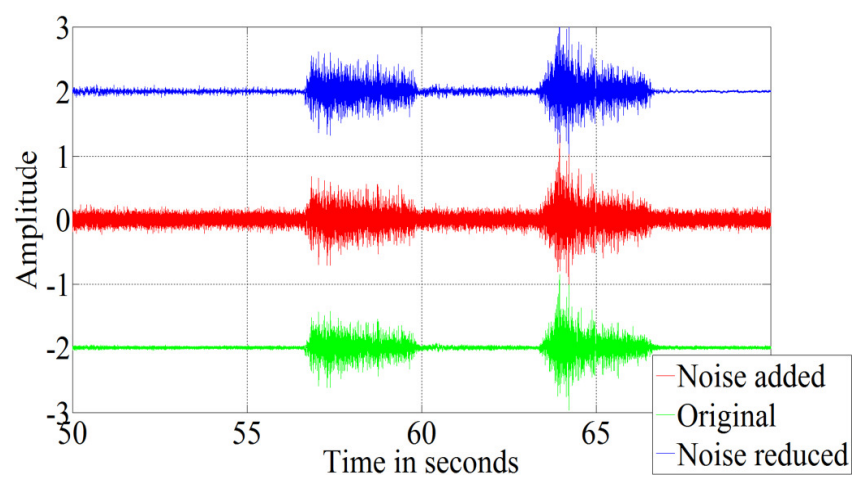

(a)

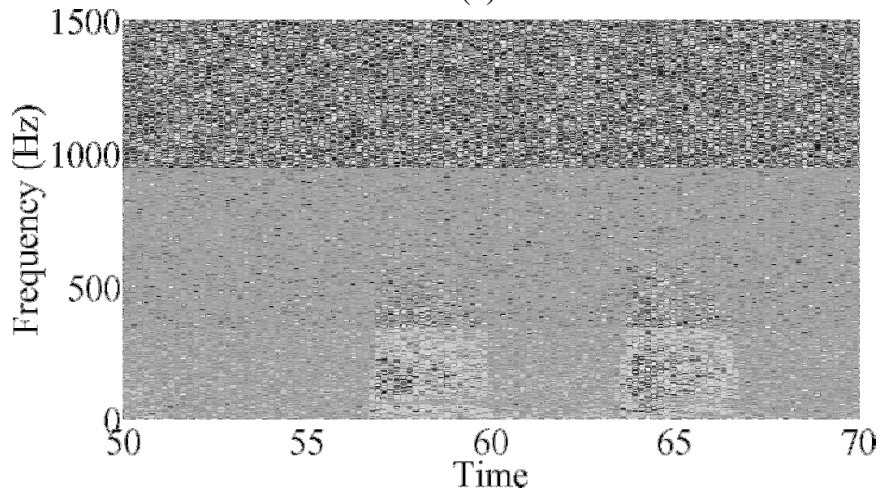

(c)

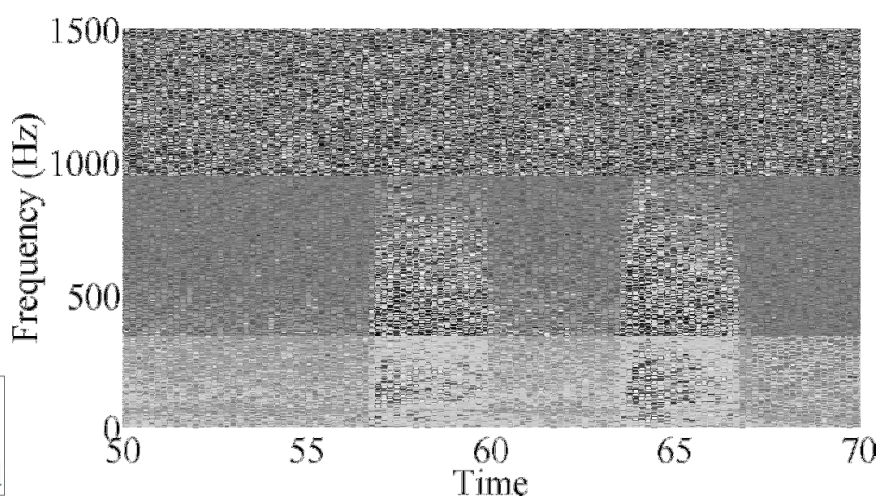

(b)

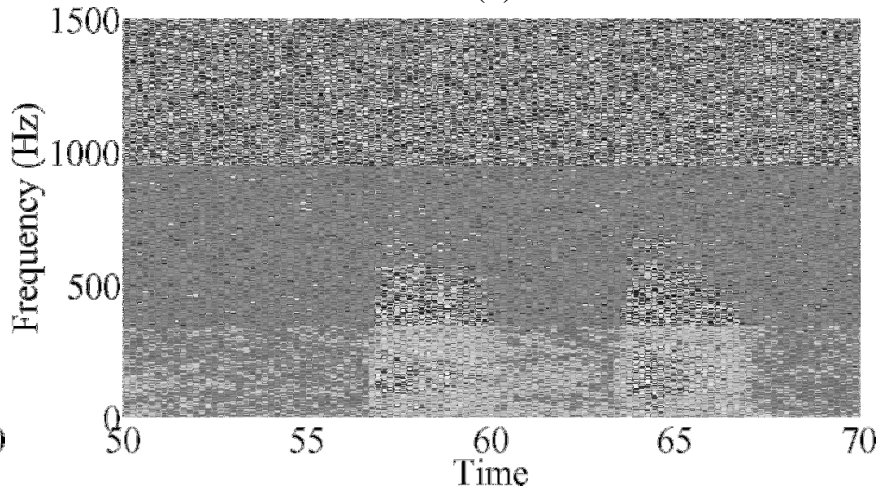

(d)

Fig. 3. Example of noise reduction using IMCRA-based spectral enhancement applied to a single channel of EMG (a) Time domain representations of channel 6. Signals have been offset on the $y$-axis for clarity - original (top), noise-added (middle), noise-reduced (bottom). The signals have been offset on the y-axis for clarity (b) spectrogram of the original signal (c) spectrogram of the signal with AWGN added (d) spectrogram of the signal after IMCRA-based spectral enhancement has been applied.

There is a trade-off between window size and number of FFT bins. Higher frequency resolution can be obtained with longer FFT windows at the expense of time resolution (and therefore response time to changing noise levels). For our purposes, human reaction time can set a rough upper limit to the window size [33].

The example in Fig. 4(a) below is of a single channel of EMG consisting of five five-second recordings separated by fivesecond inactivity periods. The signals have been vertically offset for clarity. The signal (top) and a noise-added version (middle) are shown in the time domain in Fig. 4(a). IMCRA-based spectral enhancement was applied to the noisy version of the signal (bottom).
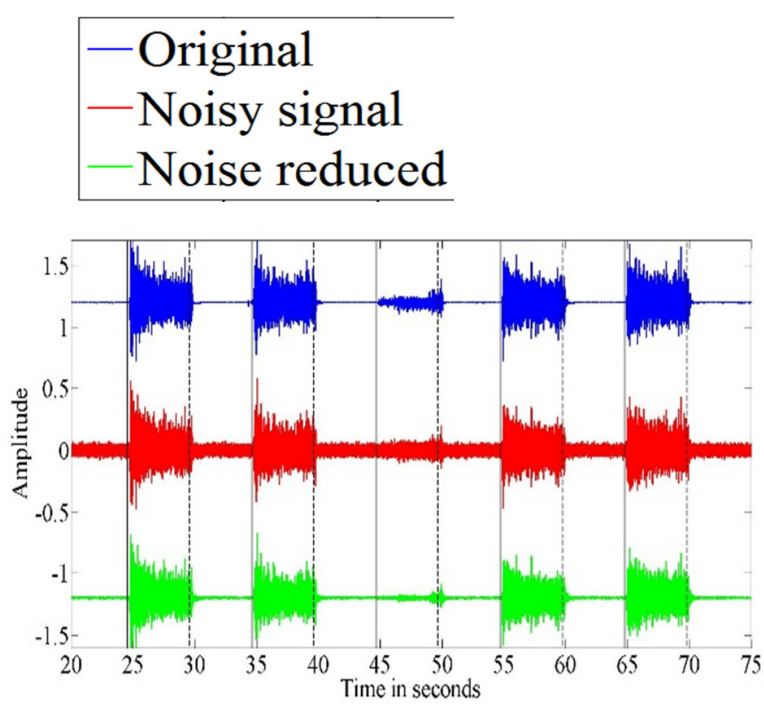

(a)
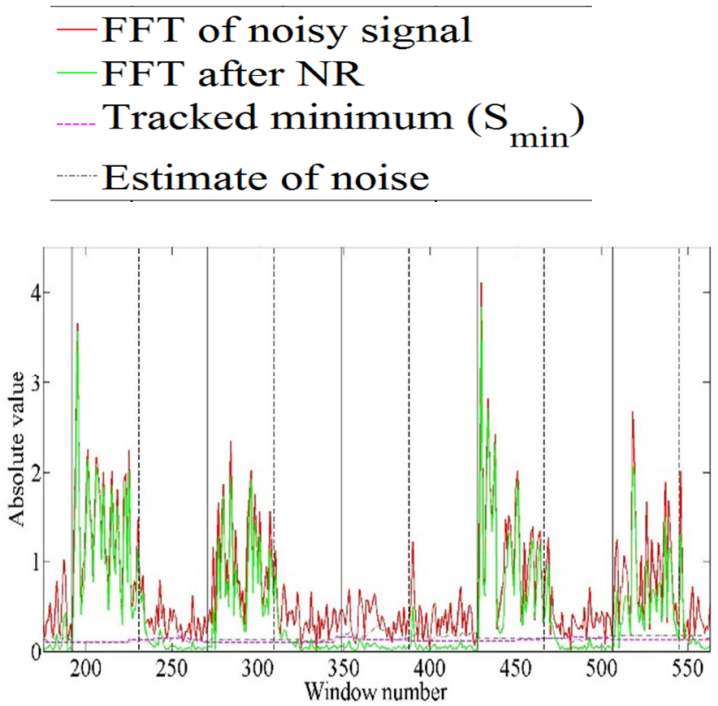

(b)

Figure 4 (a) A recording of EMG for five gestures - original signal (top), noise-added signal (middle) and noisy signal that has IMCRA-based spectral enhancement applied (bottom) offset vertically for clarity (b) magnitude of the STFT of a single bin (bin 15) along with the magnitude of the STFT for the same 
bin after the application of IMCRA-based spectral enhancement. The tracked minimum $\left(\mathrm{S}_{\min }\right)$ and the estimate of the noise are also shown

One DFT bin was chosen to demonstrate the behaviour of IMCRA upon EMG. Fig. 4(b) shows the absolute value of the STFT of bin 15 (which has a centre frequency of about $60 \mathrm{~Hz}$ ) before and after IMCRA-based spectral enhancement is applied. The timing of the instructions that were given to the subject to perform gestures and to relax are shown as solid and dashed vertical lines, respectively. It can be seen that the 'rest', or inactivity sections, which contain only noise, have been attenuated to a greater degree than the sections in which gestures are being performed.

\subsection{Comparison}

MSNE and the spectral enhancement based on IMCRA were compared against other noise reduction techniques. Three different approaches were taken. Firstly, the change in signal quality for noisy simulated EMG was tested before and after the application of each noise reduction method. Second, the change in classification accuracy was measured for Data Set 1 with added AWGN added at various levels. Finally, classification accuracy for Data Set 2 was examined for different numbers of channels with and without noise reduction. Table II lists the noise reduction methods that were compared.

TABLE II

NOISE REDUCTION METHODS TESTED

\begin{tabular}{|l|l||l|l|}
\hline A & EMG with AWGN added & I & $\begin{array}{l}\text { EMD: sum of first three } \\
\text { IMFs [25] applied to } \\
\text { signal A }\end{array}$ \\
\hline B & Band pass of signal A & J & $\begin{array}{l}\text { EMD: sum of first three } \\
\text { IMFs [25] then band pass } \\
\text { applied to signal A }\end{array}$ \\
\hline C & $\begin{array}{l}\text { IMCRA spectral } \\
\text { enhancement applied } \\
\text { signal A }\end{array}$ & K & $\begin{array}{l}\text { EMD: IMF thresholding } \\
\text { [26] applied to signal A }\end{array}$ \\
\hline D & $\begin{array}{l}\text { IMCRA spectral } \\
\text { enhancement then band } \\
\text { pass applied to signal A }\end{array}$ & L & $\begin{array}{l}\text { EMD: IMF thresholding } \\
\text { [26] then band pass } \\
\text { applied to signal A }\end{array}$ \\
\hline E & $\begin{array}{l}\text { Wavelet [17] applied to } \\
\text { signal A }\end{array}$ & M & $\begin{array}{l}\text { Bandpass of the 'clean' } \\
\text { EMG }\end{array}$ \\
\hline F & $\begin{array}{l}\text { Wavelet [17] then band } \\
\text { pass applied to signal A }\end{array}$ & N & $\begin{array}{l}\text { Wiener applied to signal } \\
\text { A }\end{array}$ \\
\hline G & $\begin{array}{l}\text { MSNE [31] applied to } \\
\text { signal A }\end{array}$ & O & $\begin{array}{l}\text { Wiener then band pass } \\
\text { applied to signal A }\end{array}$ \\
\hline H & $\begin{array}{l}\text { MSNE [31] then band } \\
\text { pass applied to signal A }\end{array}$ & & \\
\hline
\end{tabular}

In order to get the best possible improvement in classification accuracy when applying IMCRA-based spectral enhancement to Data Sets 2 and 4, it was necessary to change the values until the best possible classification accuracy was obtained for the entire data set. The values of many of the parameters given in [30] [31] were optimised for speech sampled at 16kHz, so empirical tests had to be performed to determine the best values of the parameters for EMG. The parameters were changed one at a time and then, if there was an improvement in accuracy, the best value was kept and the other values were then tested. IMCRA-based spectral enhancement was applied to each EMG channel independently. IMCRA assumes that the first window of the signal contains only noise, which is true for Data Sets 2 and 4.

The most important parameters were found to be $\mathrm{L}, \mathrm{V}$ and $\mathrm{U}$. L is the size of the STFT window in samples that was used in IMCRA, which determines (by definition) the number of FFT bins. U and V set the number of samples and frames over which the minima are tracked and updated. The values used for IMCRA with Data Sets 2 and 4, which are shown in Table III, were decided with empirical tests in which a range of values for each parameter were systematically tested in turn to assess the effect upon classification accuracy. The parameter values that were used for IMCRA are listed in Table III. These values were decided based on the highest improvements in classification accuracy obtained during empirical tests for both data sets.

TABLE III

VALUES USED FOR IMCRA SPECTRAL ENHANCEMENT

\begin{tabular}{|c|l|l|l|}
\hline Name & Description & $\begin{array}{l}\text { Value used } \\
\text { Data Set 1 }\end{array}$ & $\begin{array}{l}\text { Value used } \\
\text { Data Set 2 }\end{array}$ \\
\hline L & $\begin{array}{l}\text { Length of FFT } \\
\text { window in samples }\end{array}$ & 256 & 512 \\
\hline V & Minimum of & 15 & 10 \\
\hline
\end{tabular}




\begin{tabular}{|c|l|l|l|}
\hline & $\begin{array}{l}\text { current sub- } \\
\text { window of V } \\
\text { samples }\end{array}$ & \\
\hline $\mathrm{U}$ & $\begin{array}{l}\text { Number of sub- } \\
\text { windows within the } \\
\text { V samples for } \\
\text { minima tracking }\end{array}$ & 4 & 4 \\
\hline
\end{tabular}

The choice of statistical model for the PDF was Gaussian for EMG [34], though this is based on the assumption of constantforce, constant-angle and non-fatiguing contractions [34]. The conditional gain $G_{h}$ was used instead of $G$, therefore the presence probability was not used in the recursive smoothing of the spectral gain.

\subsubsection{Signal properties of simulated EMG}

A set of 500 dynamic simulated EMG recordings were created using the process described in Section 2.1.1, with an SNR of $0 \mathrm{~dB}$ and a single 'gesture' per simulated recording. The signal quality was measured before and after the application of each of the noise reduction methods using the criteria discussed in Section 1.

\subsubsection{Classification accuracy using real EMG with $A W G N$}

The presence of noise in EMG signals affects the classification accuracy of any pattern recognition systems that have been trained to recognise gesture by using features derived from noisy data. It is therefore asserted that, other parameters being equal, the change in classification accuracy can be used as a measure of the effectiveness of noise reduction algorithms. If a noise reduction method is effective, then a classifier trained and tested with the processed data should have a higher classification accuracy compared with a classifier that was trained and tested with features extracted from the original, unprocessed data.

For Data Set 1, a set of linear Support Vector Machines (SVMs) was used to classify gestures because LDA classifiers failed to converge at the higher noise levels. The seven lower-arm channels were used, with AWGN added at $-10 \mathrm{db},-5 \mathrm{~dB}$ and $0 \mathrm{~dB}$ SNR relative to the mean power of the gestures per channel. For feature extraction, window length was 300ms with an overlap of $150 \mathrm{~ms}$, and ten windows were taken from each gesture. For each subject, half of the data (12 of the trials) were used for training and half for testing. This was done for each trial for all thirty subjects. The mean and standard deviations of all of the accuracies across all trials of all subjects was then taken. Classification accuracy from the band passed 'clean' data was used as the reference.

\subsection{Classification accuracy using real noisy EMG}

For Data Set 2, Linear Discriminant Analysis was used for classification [2]. The gesture classification accuracies for all combinations of two channels out of sixteen were calculated, and then the mean across all combinations for all five subjects was taken. This was repeated for four, six, eight, ten, twelve and fourteen channel combinations for each of the processes tested, for the purposes of comparing noise reduction against the effectiveness of adding more channels. The mean accuracy across all subjects when all sixteen channels were used was assessed for each process and used as the reference.

\section{RESUlts}

\subsection{Signal properties of simulated EMG}

The properties of the simulated EMG that was contaminated with AWGN at OdB were investigated before and after each of the noise reduction techniques. The results varied greatly depending on whether band pass filtering was also used. For this reason, results both with and without band pass filtering are given below. The SNR, DP Ratio, SN Ratio and $\Omega$ Ratio before and after noise reduction were investigated using the method described in 3.1.1. The results are shown in Fig. 5. 


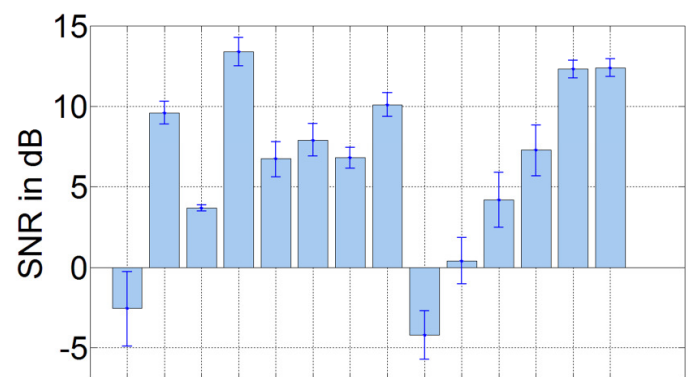

A B C D E $\underset{\text { Process label }}{\mathrm{F}} \mathrm{H}$ I $\mathrm{J} \mathrm{L} \mathrm{M} \mathrm{N}$

(a)

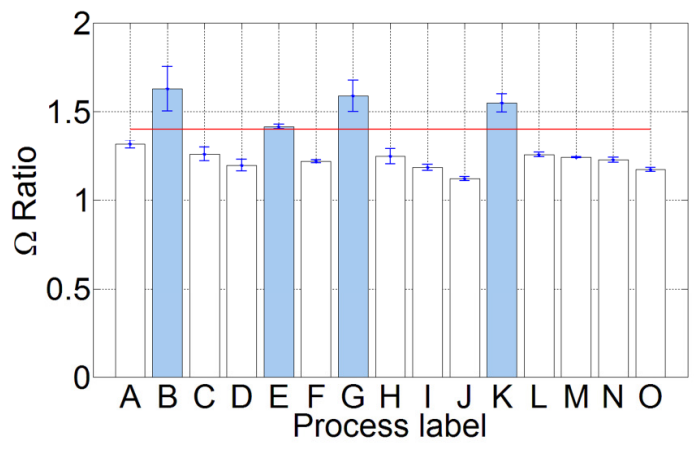

(c)

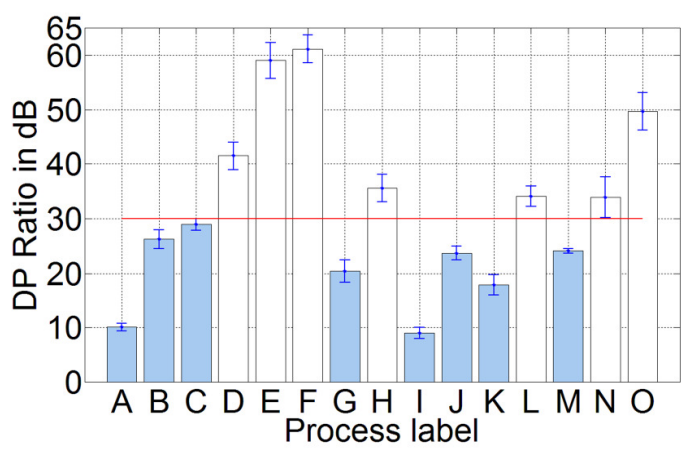

(b)

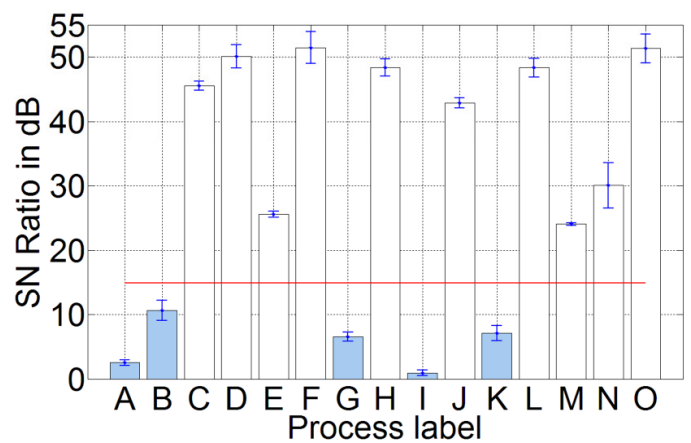

(d)

Fig. 5. Mean change in signal properties for each of the filtering types across the 500 simulated gestures with AWGN added at 0dB SNR. (a) SNR (b) DP ratios horizontal line is minimum DP Ratio specified in [15] (c) $\Omega$ Ratio - horizontal line is minimum $\Omega$ Ratio specified in [15] (d) SN Ratio - horizontal line is SN Ratio for clean signal. Note that lower is better for $\Omega$ Ratio. For (b)-(d), adequate performance is highlighted in white. Table II lists the process labels.

Fig. 5 shows that Wiener ( $\mathrm{N}$ and $\mathrm{O}$, with and without band pass respectively) has the most consistent improvement in all criteria compared with the other methods. However, Wiener filtering requires a clean reference, which is available for this simulated data set, but will be unavailable in a real EMG-based control system. The results obtained using the IMCRA-based spectral enhancement along with band passing adequately corrected the DP Ratio, $\Omega$ Ratio and SN Ratio. Fig. 4(d) shows that band passing the signal regardless of the processing method improves the $\Omega$ Ratio.

\subsection{Classification accuracy using Data Set 1 with added AWGN}

The classification accuracy was investigated using the method described in Section 3.1.2 applied to Data Set 1 with Additive White Gaussian Noise added at three different SNRs. The classification accuracy results are shown in Fig. 6, where the process labels are defined in Table II.

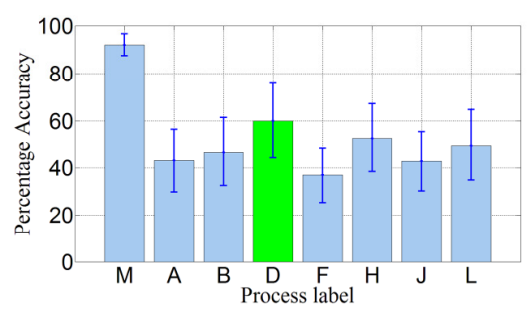

(a)

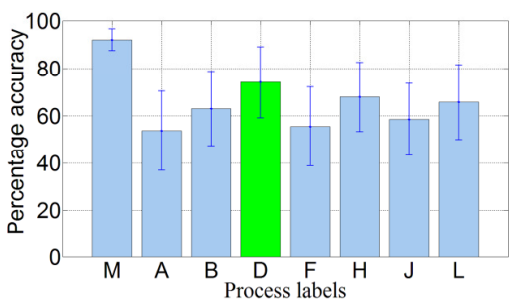

(b)

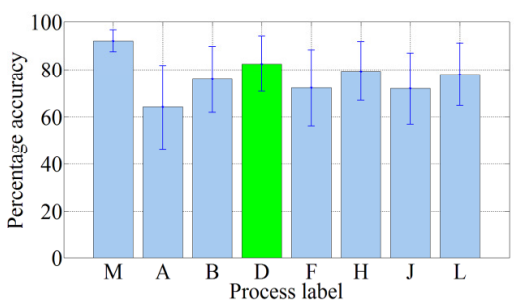

(c)

Fig. 6. Mean accuracies across all subjects of the Data Set 1 for all processes with AWGN added (a) at $-10 \mathrm{~dB}$, (b) at $-5 \mathrm{~dB}$, (c) at $0 \mathrm{~dB}$ relative to the mean power of the 'gesture' parts of each of the signals. The error bars are \pm 1 SD. Processes labels are defined in Table II. IMCRA with bandpass is highlighted.

The same results are shown in Table III below.

TABLE III

MEAN ACCURACIES ACROSS ALL SUBJECTS OF THE DATA SET 1 FOR ALL PROCESSES WITH AWGN ADDED AT -10DB, -5DB, 0DB RELATIVE TO THE MEAN POWER OF THE 'GESTURE’ PARTS OF EACH OF THE SIGNALS 


\begin{tabular}{|l|r|l|l|l|l|l|l|l|}
\hline -10dB & M & A & B & D & F & H & J & L \\
\hline Mean & 92.01 & 43.02 & 46.87 & 59.90 & 36.83 & 52.75 & 42.73 & 49.69 \\
\hline SD & 4.60 & 13.45 & 14.50 & 15.78 & 11.76 & 14.37 & 12.71 & 15.04 \\
\hline & & & & & & & & \\
\hline -5dB & M & A & B & D & F & H & J & L \\
\hline Mean & \multicolumn{1}{|r|}{92.01} & 53.66 & 63.00 & 74.08 & 55.43 & 67.93 & 58.41 & 65.74 \\
\hline SD & 4.60 & 17.09 & 16.76 & 14.25 & 17.05 & 14.98 & 15.64 & 15.80 \\
\hline & & & & & & & & \\
\hline OdB & M & A & B & D & F & H & J & L \\
\hline Mean & 92.01 & 64.06 & 75.79 & 82.35 & 72.13 & 79.30 & 71.85 & 77.97 \\
\hline SD & 4.60 & 17.59 & 13.91 & 11.63 & 16.03 & 12.38 & 15.03 & 13.19 \\
\hline
\end{tabular}

In all parts of Fig. 6 and Table III, bar 'M' shows the accuracy for the unfiltered clean data, which is $92 \%$. Fig. 5 (a) show the results for noise added at $-10 d B$ relative to the mean gesture power, where the accuracy ' $A$ ' (signal with AWGN added) is $43 \%$. The IMCRA-based spectral enhancement (D) is best at restoring the accuracy to $59.9 \%$ with a standard deviation of 15.8 . There is a similar trend in improvement at $-5 \mathrm{~dB}$ in Fig. 6 (b) and at $0 \mathrm{~dB}$ in Fig. 6 (c).

\subsection{Classification accuracy using real noisy EMG}

Data Set 2 has high levels of power line interference, so notch filtering is applied along with band pass filtering before noise reduction is applied. Classification accuracy for Data Set 2 (shown in Fig. 7) is high for all five subjects when all sixteen channels are used after band pass and notch filtering.

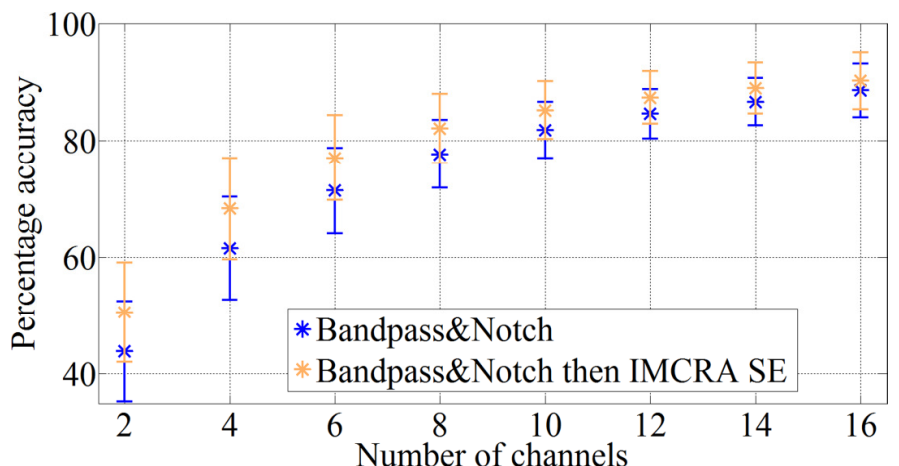

Fig. 7. Mean accuracies across all five subjects across all combinations of 2, 4, 6, 8, 10,12, 14 and 16 channels of Data Set 2 . The results with and without IMCRA-based spectral enhancement (shown as IMCRA-based spectral enhancement) are shown. The error bars are \pm 1 standard deviation

In Fig. 7, as could be expected, the accuracy drops as fewer channels are used. The drop-off is less prominent if IMCRA-based spectral enhancement is applied, and the improvement is greater for fewer channels. For example, for two channels, the accuracy jumps from a mean of $43.9 \%$ to $50.6 \%$. For four channels, the mean accuracy jumps from $61.5 \%$ to $68.3 \%$. For sixteen channels, the mean accuracy across all subjects increases slightly from $88.58 \%$ to $90.2 \%$.

The mean improvements range between $1.5 \times$ a standard deviation for two channels to $1.3 \times$ a standard deviation for 16 channels.

\section{DISCUSSION}

Fig. 5 shows that the measurements of signal quality found in [15] are affected in different ways by each of the methods, but that IMCRA-based spectral enhancement restores the values to within the acceptable ranges given in the reference. Fig. 6 shows that it is the most effective of the algorithms at recovering the classification accuracy in the presence of AWGN. Confidence that noise is being removed is increased by the observation that the classification accuracy of the pattern recognition systems is improved. Fig. 7 shows some classification accuracy can be recovered for noisy real EMG when fewer channels are used.

IMCRA is sensitive to initial conditions: it is important that the first window consist only of noise. A test was performed in which the noise estimation started during a gesture. The IMCRA estimator assumed this to be the noise floor, so the rest of the signal was attenuated accordingly, which rendered the signals useless for pattern recognition purposes.

Some tests were carried out to assess the effectiveness of IMCRA on Data Set 1 for varying numbers of channels. The results from Data Set 1 changed only by a little either way if IMCRA-based Spectral Enhancement was applied to the 'clean' signals. 
This indicated that the noise estimation was good. The length of the window (L) is determined by the need for the classifier to respond within human reaction time. Changing L to 1024 for the real EMG increased the accuracy slightly for Data Set 1, but this is longer than the $300 \mathrm{~ms}$ reaction time.

To determine the effects of varying parameters, the values of the smoothing factors are critical. The time-varying value of $\alpha$, $\hat{\alpha}$, depends on the conditional speech presence probability $p$. $\hat{\alpha}$ determines the trade-off between signal attenuation and noise reduction. $\alpha_{\mathrm{d}}$ and $\alpha_{\mathrm{s}}$ are also smoothing factors, the values of which determine the smoothness of the recursive spectral estimation for the noise and minima spectrum respectively [31]. The forgetting factor, $\alpha$, a, must not be too low or the spectral peaks caused by gestures will be insufficiently strong.

With the Data Set 2, there were diminishing returns on the use of IMCRA-based spectral enhancement as more channels were used. Increasing the number of EMG channels was found to improve the classification accuracy even for very noisy data. If there are fewer channels, then IMCRA-based spectral enhancement provides a significant improvement in accuracy.

The results have shown that band passing of the signal should always be performed regardless of the noise reduction type employed.

\section{CONCLUSION}

This paper has shown the potential of spectral noise estimation and noise reduction for improving the classification accuracy of a pattern recognition-based myoelectric control system. The change in signal quality in the presence of AWGN was examined and found to be satisfactory. Two approaches were then used to demonstrate that Spectral Enhancement techniques such as IMCRA can improve myoelectric signal quality and therefore classification accuracy for the purpose of pattern recognitionbased prosthetic hand control. This implies that applying the technique based on IMCRA is more effective than the other noise reduction methods at preserving spectral information from the EMG while reducing the noise.

The improvement in accuracy gained for the noisy data would make the operation of prosthetic hands easier. IMCRA was seldom found to be detrimental to classification accuracy if performed on data that was already clean, but there is of course additional processing required for the spectral noise reduction.

\section{ACKNOWLEDGMENT}

The authors would like to thank Prof Adrian D. C. Chan of Carleton University, Ottawa for advice and for providing the Data Set 1. The authors also thank Dr Christian Cipriani of ARTS Lab, Italy for providing Data Set 2.

\section{REFERENCES}

[1] N. H. Lovell et al., "Biosignal quality detection: An essential feature for unsupervised telehealth applications," IEEE Intl Conf e-Health Networking Appls Services (Healthcom), 81-85, 2010.

[2] B. Hudgins, P. Parker, R. N. Scott., "A new strategy for multifunction myoelectric control”, IEEE Transactions on Biomedical Engineering Vol. 40 No. 1, pp. 82-94, 1993

[3] Mohammadreza Asghari Oskoei, Huosheng Hu, "Myoelectric control systems: a survey", Biomedical Signal Processing and Control 2, 2007 , pp. 375-294.

[4] Paul McCool, Graham D. Fraser, Adrian D. C. Chan, Lykourgos Petropoulakis, John J. Soraghan, "Identification of Contaminant Type in Surface Electromyography (EMG) Signals", submitted to IEEE Transactions on Neural Systems and Rehabilitation Engineering

[5] G. D. Fraser, A. D. C. Chan, J. R. Green, N. Abser, D. MacIsaac , "CleanEMG - Power line interference estimation in sEMG using an adaptive least squares algorithm", 33rd Annual International Conference of the IEEE EMBS, Boston, Massachusetts USA, August 30 - September 3, 2011, pp. 79417944.

[6] Paul McCool, Graham D. Fraser, Adrian D. C. Chan, Lykourgos Petropoulakis, John J. Soraghan, "Identification of Contaminant Type in Surface Electromyography (EMG) Signals", IEEE Transactions on Neural Systems and Rehabilitation Engineering, in press.

[7] G. Fraser, A. D. C. Chan, J. R. Green, D. MacIsaac, "Automated biosignal quality analysis for electromyography using a one-class support vector machine," submitted to IEEE Transactions on Instrumentation and Measurement, 2013.

[8] Edward A. Clancy and Kristin A. Farry, "Adaptive Whitening of the Electromyogram to Improve Amplitude Estimation", IEEE Transactions on Biomedical Engineering, Vol. 47, No. 6, June 2000.

[9] D. P. Allen, "A frequency domain Hampel filter for blind rejection of sinusoidal interference from electromyograms", Journal of Neuroscience Methods, 2009, Vol. 177 NO. 2, pp. 303-10. doi:10.1016/j.jneumeth.2008.10.019.

[10] D. T. Mewett, K.J. Reynolds, H. Nazeran "Reducing power line interference in digitised electromyogram recordings by spectrum interpolation", Medical \& Biological Engineering \& Computing. Vol. 42 No. 4, pp. 524-31.

[11] G. D. Fraser, A. D. C. Chan, J.R. Green, D. MacIsaac, "Removal of electrocardiogram artifacts in surface electromyography using a moving average method," in Medical Measurements and Applications Proceedings (MeMeA), 2010 IEEE International Symposium on, 2012, pp. 128-131.

[12] Angkoon Phinyomark, Chusak Limsakul, and Pornchai Phukpattaranont, "A Novel Feature Extraction for Robust EMG Pattern Recognition", Journal of Computing 1, Vol 1, December 2009, pp. 71-80

[13] Angkoon Phinyomark, Chusak Limsakul, Pornchai Phukpattaranont "A Comparative Study of Wavelet Denoising for Multifunction Myoelectric Control", International Conference on Computer and Automation Engineering, 2009, pp. 21-25.

[14] Xiaomei Ren, Zhiguo Yan, Zhizhong Wang, Xiao Hu, "Noise reduction based on ICA decomposition and wavelet transform for the extraction of motor unit action potentials", Journal of Neuroscience Methods 158, 2006, pp. 313-322.

[15] C Sinderby, L Lindstrom, A.E. Grassino, “Automatic assessment of electromyogram quality”, J Appl Physiol 79: 1803-1815, 1995

[16] R. Merletti, "Standards for reporting EMG data", Journal of electrophysiology and kinesiology 9(1):III-IV, February 1999, available www.isekonline.org/standards_emg.html, accessed August 2014.

[17] D. K. Kumar, S. Poosapadi Arjunan, V. P. Singh, "Towards identification of finger flexions using single channel surface electromyography--able bodied and amputee subjects," Journal of neuroengineering and rehabilitation, vol. 10, p. 50, 2013. 
[18] Roberto Merletti, Philip Parke, "Electromyography: Physiology, Engineering, and Noninvasive Applications”, John Wiley \& Sons, 2004 , pp. $205-207$.

[19] Edward -Shwedyk, R. Balasubramanian, R. N. Scott, “A Nonstationary Model for the Electromyogram, IEEE Trans. On Biomed Eng, Vol. BME-24, No. 5, Sep 1977, pp. 417-424

[20] Gerhard Staude, Claus Flachenecker, Martin Daumer, Werner Wolf, “Onset Detection in Surface Electromyographic Signals: A Systematic Comparison of Methods”, EURASIP Journal on Applied Signal Processing 2001:2, pp. 67-81.

[21] A. D. C Chan and G. C. Green, "Myoelectric control development toolbox," presented at the 30th Conf. Can. Med. Biol. Eng. Soc., Toronto, Canada, 2007, Paper M0100.

[22] H. Daley, K. Englehart, L. Hargrove, U. Kuruganti, "High density electromyography data of normally limbed and transradial amputee subjects for multifunction prosthetic control", J Electromyogr Kinesiol. 2012 Jun;22(3):478-84. Epub 2012 Jan 24.

[23] Norbert Wiener, "The extrapolation Interpolation and Smoothing of Stationary Time Series with Engineering Applications", J. American Statistical Association, Vol. 47, No. 258, 1952, pp. 319-321.

[24] Norden E. Huang, Zheng Shen, Steven R. Long, Manli C. Wu, Hsing H. Shih, Quanan Zheng, Nai-Chyuan Yen, Chi Chao Tung, Henry H. Liu, "The empirical mode decomposition and the Hilbert spectrum for nonlinear and non-stationary time series analysis”, Proc. R Soc. Lond. A (1998) 454, pp. 905995.

[25] Joyce Chiang, Z. Jane Wang, Martin J. McKeown, ”A Hidden Markov, Multivariate Autoregressive (HMM-mAR) Network Framework for Analysis of Surface EMG (sEMG) Data", IEEE Trans. On Signal Processing, vol. 56, No. 8 2008, pp. 4069-4081.

[26] Abdel-Ouahab Boudraa, Jean-Christophe Cexus, "EMD-based signal filtering", IEEE Trans. on instrumentation and measurement, Vol. 56, No. 6, Dec 2007

[27] C. Castellini, E. Gruppioni, A. Davalli, and G. Sandini, "Fine detection of grasp force and posture by amputees via surface electromyography," J Physiol Paris, 10 April 2009.

[28] M. Berouti., M. Schwartz, J. Makhoul, “Enhancement of Speech corrupted by acoustic noise”, IEEE Conference on Acoustics, Speech and Signal Processing (ICASSP), 1979. 208-211.

[29] Rainer Martin, "Spectral Subtraction based on Minimum Statistics”, EUSIPCO 1994, pp. 1182-85

[30] Israel Cohen, "Noise spectrum estimation in adverse environments: improved minima controlled recursive averaging", IEEE Transactions on Speech and Audio Processing, Vol. 11 No. 5, September 2003

[31] Jacob Benesty, M. Mohan Sondhi, Yiteng Huang (Eds.), "Springer handbook of speech processing", Springer 2008, e-ISBN: 978-3-540-49127-9

[32] N. Chatlani and J. J. Soraghan, "EMD-Based Filtering (EMDF) of Low-Frequency Noise for Speech Enhancement," Audio, Speech, and Language Processing, IEEE Transactions on, vol. 20, pp. 1158-1166, 2012.

[33] Adrian D. C. Chan and Kevin B. Englehart, "Continuous Myoelectric Control for Powered Prostheses Using Hidden Markov Models", IEEE transactions on biomedical engineering, Vol. 52, No. 1, Jan 2005.

[34] E. A. Clancy, N. Hogan, "Probability density of the surface electromyogram and its relation to amplitude detectors", IEEE Trans. Biomed. Eng., vol. 46, no. 6, pp. 730-739, Jun. 1999

Paul McCool Paul McCool received B.Eng. (Hons.) degree in electronic engineering and physics from the University of Glasgow, Glasgow, U.K., in 1999, and the M.Sc. degree (with distinction) in electronic and electrical engineering, in 2010, from the University of Strathclyde, Glasgow, U.K., where from 2010-2014 he read a Ph.D. degree researching pattern recognition and muscle activity detection for forearm myoelectric signals to control a prosthetic hand.

$\mathrm{He}$ is currently a Research Associate at Heriot-Watt University, where he is applying pattern recognition and image processing to medical images to assist with diagnosis.

He worked as a Project Officer in the Defence Equipment and Support agency of the U.K. Ministry of Defence between 1999 and 2009.

Mr. McCool is a member of the IET.

Lykourgos Petropoulakis was born in Athens, Greece. He obtained a first class degree in Aeronautical Engineering and a PhD in Control Engineering from the University of Salford, UK. Subsequently, he joined Edinburgh University where he worked as a researcher in artificial intelligence and robotics. In 1992 he accepted a lectureship position in the Department of Electronic and Electrical Engineering in the University of Strathclyde. Dr. Petropoulakis has over 80 publications in journals and conferences. His interests are currently in robotics, microprocessors, prosthetic devices, intelligent systems and bioengineering.

John J. Soraghan (S'83-M'84-SM'96) received the B.Eng. (Hons.) and M.Eng.Sc. degrees in electronic engineering from University College Dublin, Dublin, Ireland, in 1978 and 1983, respectively, and the Ph.D. degree in electronic engineering from the University of Southampton, Southampton, UK, in 1989. His doctoral research focused on synthetic aperture radar processing on the distributed array processor.

After graduating, he worked with the Electricity Supply Board in Ireland and with Westinghouse Electric Corporation in the U.S. In 1986, he joined the Department of Electronic and Electrical Engineering, University of Strathclyde, Glasgow, UK, as a Lecturer and became a Senior Lecturer in 1990, a Reader in 2000, and a Professor in signal processing in September 2003. He was a Manager of the Scottish Transputer Centre from 1988 to 1991 , Manager with the DTI Parallel Signal Processing Centre from 1991 to 1995 and Head of the ICSP from 2005-2007. He currently holds the Texas Instruments Chair in signal processing within the Centre for excellence in Signal and Image Processing (CeSIP), University of Strathclyde. His main research interests are signal processing theories, algorithms, and architectures with applications to high resolution methods for radar and acoustics, biomedical data processing, video analytics for surveillance, $3 \mathrm{D}$ video and condition monitoring.

Professor Soraghan is a member of the IEEE Signal Processing in Education Technical Committee, a Member of the IET and a Senior Member of the IEEE.

Navin Chatlani received the B.Sc. (Hons.) degree in electrical and computer engineering from the University of the West Indies, Trinidad, in 2002 and the M.Sc. (with distinction) and Ph.D. degrees in electronic and electrical engineering from the University of Strathclyde, Glasgow, U.K., in 2007 and 2011, respectively. His doctoral research focused on advanced signal enhancement techniques with application to speech and hearing.

From October 2010 to March 2012 he has was a Postdoctoral Research Fellow at the Centre for Excellence in Signal and Image Processing, University of Strathclyde, investigating methods for noise reduction, voice activity detection, beamforming, and event onset detection. Currently he works with the Audio R\&D team at Intel developing novel techniques for speech processing technologies. His main research interests are signal processing theories, algorithms, architectures, and filtering techniques for speech/audio applications and biomedical data applications. 